\title{
Histopathogenesis of intestinal metaplasia: minute lesions of intestinal metaplasia in ulcerated stomachs
}

\author{
K MUKAWA, T NAKAMURA, G NAKANO, Y NAGAMACHI \\ From the First Department of Surgery, Gunma University School of Medicine, Maebashi, Japan
}

SUMMARY Minute lesions of intestinal metaplasia composed of a few metaplastic tubules were observed in the gastric mucosa during routine histological examination of gastrectomy specimens. The histological findings indicated that these lesions might be an initial stage of more advanced intestinal metaplasia. Accordingly, more than 18000 serial sections in 10 stomachs with chronic ulcers were examined to clarify the histopathogenesis of the intestinal metaplasia. It was concluded from the three dimensional reconstruction of minute intestinal metaplasia lesions that these lesions originated during the regenerative process of healing of gastric erosions. The lesions were roughly globoid with a depression on the surface. It is thought that with continuous formation and healing of gastric erosions, more extensive intestinal metaplasia lesions would be formed by an increase in size and confluence of these focal minute intestinal metaplasia lesions.

Although theories regarding the histogenesis of intestinal metaplasia have been published by many investigators, controversy still exists. Moszkowicz mentioned that intestinal metaplasia originated from undifferentiated regenerative epithelium in the initial stages of ulcer healing. ${ }^{1}$ Oohara also recently reported that intestinal metaplasia commonly occurred as a regenerative process in ulcers. ${ }^{2}$ On the other hand, Murakami and Oohara proposed that intestinal metaplasia occurred through the transformation of proliferative cells in the necks of gastric tubules. ${ }^{34}$ Moreover, Hattori studied small carcinomas and concluded that intestinal metaplasia, dysplasia, and adenocarcinoma can arise coincidentally in the proliferative cell zone in the neck region. ${ }^{5} \mathrm{We}$, however, have rarely seen the initial stage of a minute intestinal metaplasia lesion confined to the neck region of the tubules. Minute intestinal metaplasia lesions measuring less than $1.5 \mathrm{~mm}$ in diameter, which were regarded as an initial stage of intestinal metaplasia, showed a depression on the surface. In the adjacent mucosa clusters of regenerative epithelial cells were observed and the muscularis mucosae was split or thickened by fibrosis, or both. There was an infiltrate of lymphocytes around the lesions. These histological findings indicate that focal intestinal

Accepted for publication 21 July 1986 metaplasia is derived from the repair process of gastric erosions. From these findings it was concluded that more extensive intestinal metaplasia lesions would be formed by an increase and confluence of these focal minute intestinal metaplasia lesions. Accordingly, we decided to observe smaller intestinal metaplasia lesions in greater detail to elucidate their histogenesis, by examining the three dimensional structure of the lesions and the adjacent mucosa, using histological sections.

\section{Material and methods}

Ten stomachs in which there was mild intestinal metaplasia were selected from 66 stomachs from patients with peptic ulcers which had been resected between 1979 and 1985 at this hospital. Of these 10 stomachs, four had been resected for stomach ulcer, four for duodenal ulcer, and two for gastroduodenal ulcer. The stomachs were fixed in $10 \%$ formalin. The gastric mucosa, including the ulcers, was cut into $5 \mathrm{~mm}$ thick rectangular blocks and all of these were processed and embedded in paraffin wax. Five micrometer sections were cut and stained with haematoxylin and eosin and were examined for minute intestinal metaplasia lesions. More than 18000 serial sections were made from the 35 blocks in which minute intestinal metaplasia lesions were found, to study their three dimensional structure. In this way we were able to define 


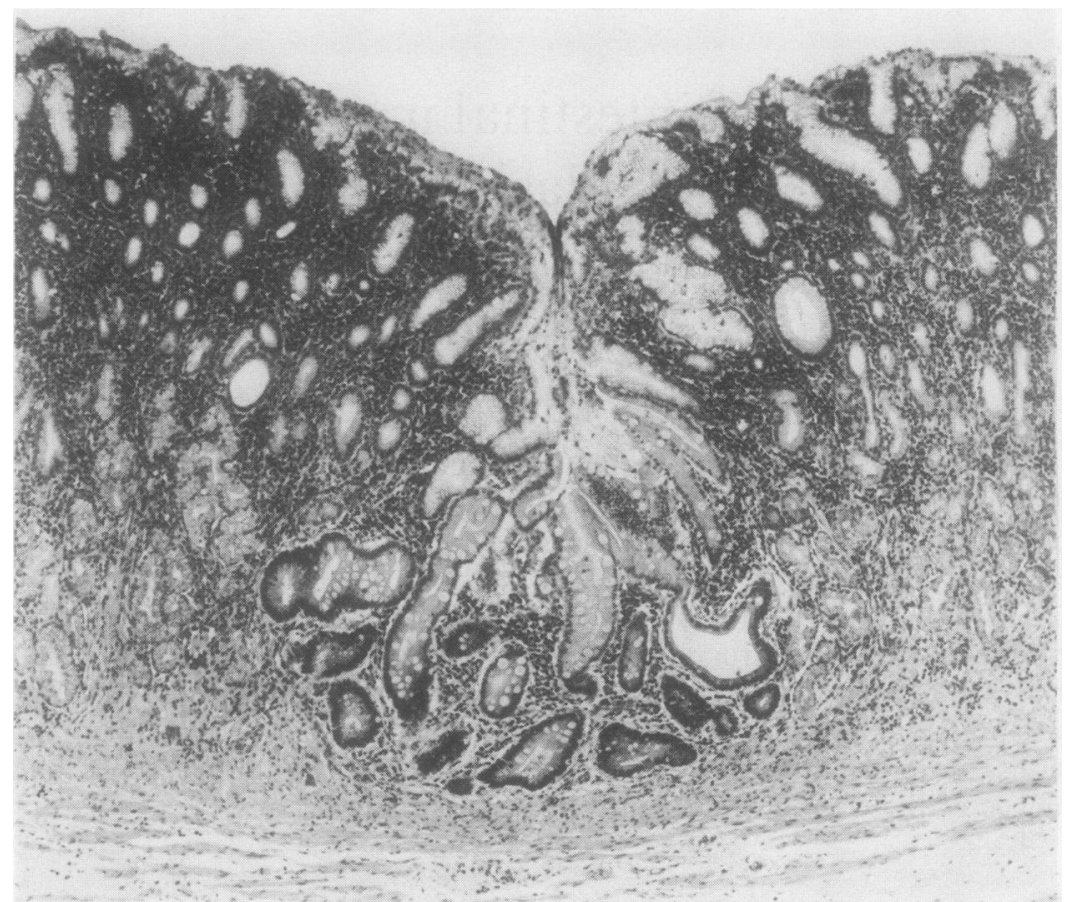

Fig 1 Typical minute intestinal metaplasia lesion composed of slight depression on surface in intermediate zone. Original magnification $\times 32$.

their form, that of the neighbouring mucosa, and to measure them three dimensionally. In defining the characteristic feature of minute intestinal metaplasia lesions as "minute" we included only those lesions measuring less than $1.5 \mathrm{~mm}$.

\section{Results}

In the 10 stomachs studied we found and reconstructed 52 minute intestinal metaplasia lesions three dimensionally from more than 18000 serial sections. Fig 1 shows a typical example of these lesions arising in the intermediate zone of the mucosa. These foci were seen randomly in the stomachs and had no specific distribution. The average age of all subjects was 45.6 years.

Fig 2a shows how metaplastic tubules began to appear in the left sections (1-4), increased in number of the upper and middle sections (5-8), and gradually disappeared in the lower sections (9-12). Microscopic three dimensional morphology showed that this lesion was roughly globoid with a dimple on the surface and was $0.55 \mathrm{~mm}$ wide and $0.75 \mathrm{~mm}$ thick. Fig $2 \mathrm{~b}$ shows an enlargement of part of fig $2 a$ (black rectangle). A noticeable decrease in the pyloric gland was observed at the centre of the minute intestinal metaplasia lesion. The neighbouring mucosa showed no intestinal metaplasia at all, and was composed of hyperplastic foveolar epithelium. The muscularis mucosae was split and thickened by fibrosis at the base. Hyperplasia of lymphoid tissue was seen in the adjacent mucosa. The metaplasia was of the complete type and included Paneth cells. Fig $3 \mathrm{a}$ is a complete serial section of another, smaller lesion which was composed of a few metaplastic tubules and measured $0.52 \mathrm{~mm}$ wide and $0.66 \mathrm{~mm}$ thick. Fig $3 \mathrm{~b}$ shows an enlargement of the photomicrograph within the black frame rectangle. The clusters of regenerative foveolar epithelial cells were clearly seen on both sides with a considerable decrease in size of the pyloric glands at the centre of the lesion. Hyperplasia of lymphoid tissue and splitting and fibrous thickening of the muscularis mucosae was also observed. A minute intestinal metaplasia lesion was seen adjacent to the erosion in the process of repair. The opening of the tubules showed nests of epithelial cells, which showed transition from regenerative undifferentiated epithelium to intestinal epithelium. The intestinal metaplasia was the complete type, including Paneth cells.

Fig 4 also shows a minute intestinal metaplasia lesion with typical foci of regenerative undiffer- 

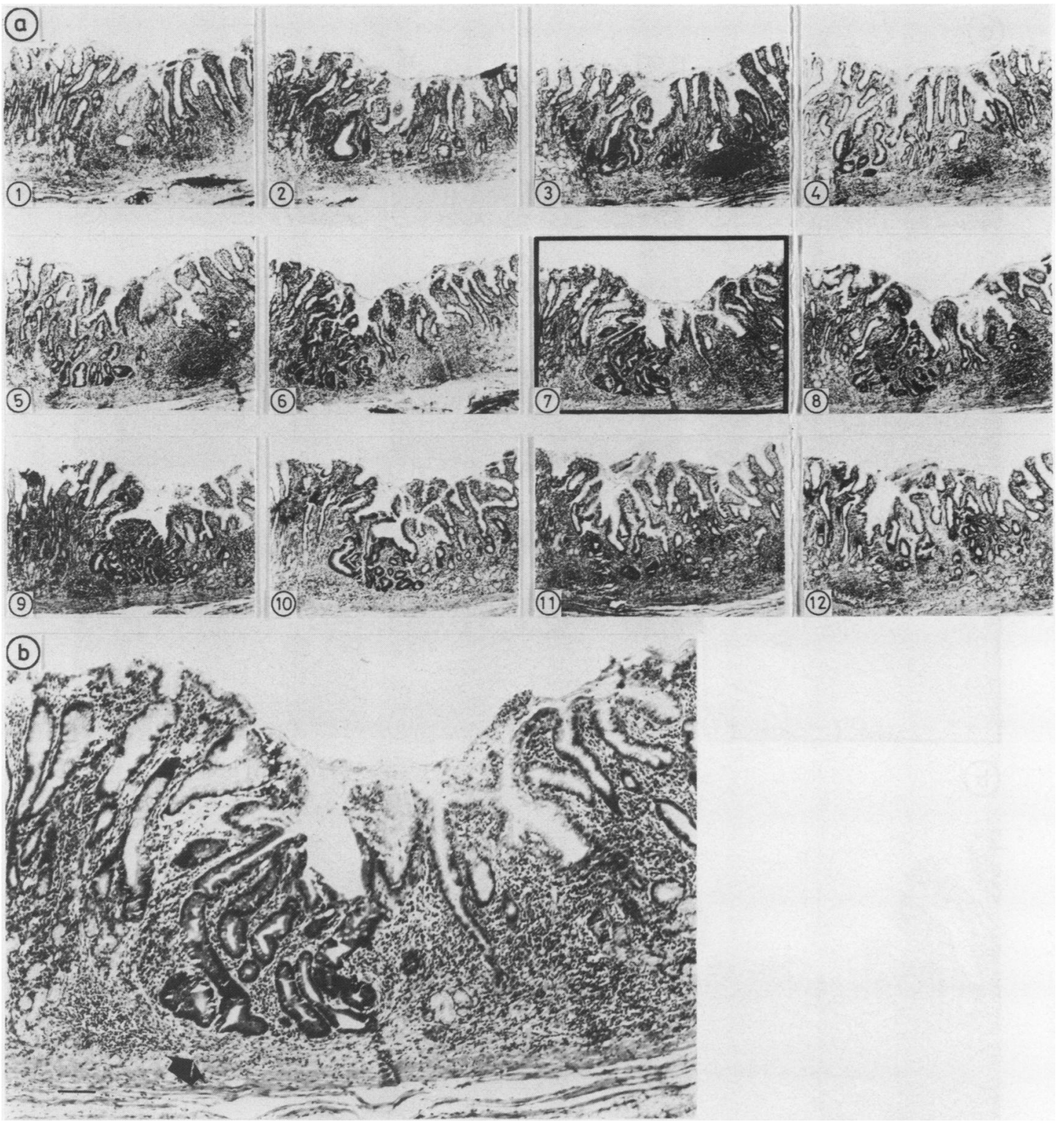

Fig 2 Complete serial sections of minute intestinal metaplasia lesion. (a) Photomicrographs show advent of and early increase in number of metaplastic tubules (top row) and then their gradual disappearance (bottom row). This lesion was roughly globoid with a depression on surface. (b) Enlargement of photomicrograph in black frame. Arrow points to minute intestinal metaplasia lesion. Considerable decrease in pseudopyloric glands with proliferation of lymphoid tissue. Original magnification $\times 32$. Bar $=200 \mu \mathrm{m}$.

entiated epithelium. There was a nest of metaplastic tubules that suggested transition from the regenerative undifferentiated epithelium to intestinal epithelium towards the base of the lamina propria. The term "regenerative undifferentiated epithelium" is used in this study to mean hyperplastic epithelium with a syncytium like appearance, which is not yet differentiated to gastric epithelium or intestinal epithelium. The epithelial cells were "flattened" or cuboidal, had partial or complete loss of the normal mucin content, and had decreased amounts of cytoplasm with an increase of basophilia. Their limiting 

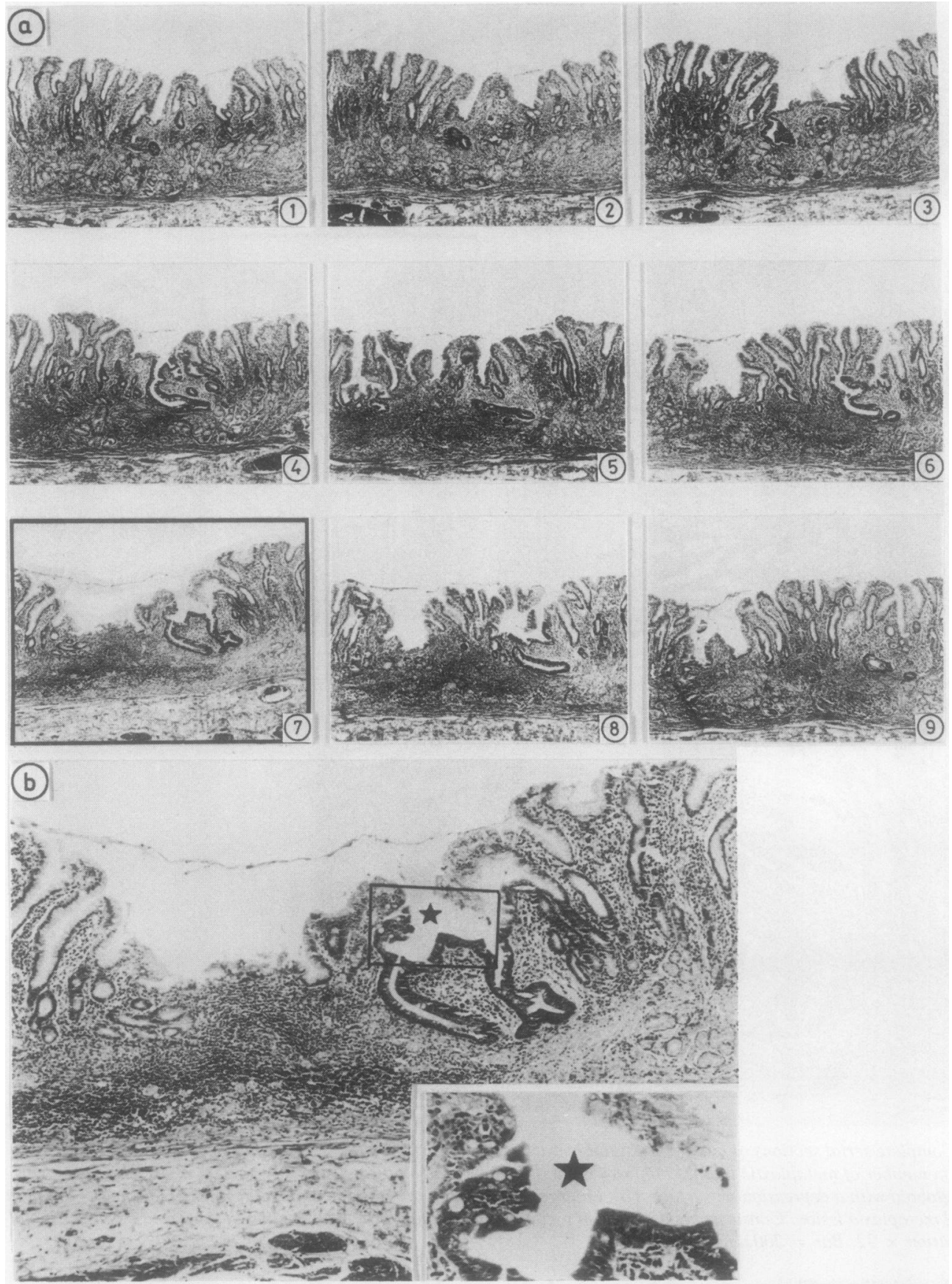

Fig 3 Minute intestinal metaplasia lesion coexisting with repairing erosion and regenerative undifferentiated epithelium. (a) Photomicrographs show advent of and early increase in size of lesion (1-7) and its gradual disappearance (8-9). (b) Enlargement of photomicrograph in black frame. Minute intestinal metaplasia lesion is seen in repairing mucosa with considerable decrease in size of pseudopyloric tubules, lymphoid hyperplasia, and thickening of muscularis mucosae. Original magnification $\times 20$. Insert shows high power view of regenerative undifferentiated epithelium $\left(^{*}\right)$. Original magnification $\times 40$. 


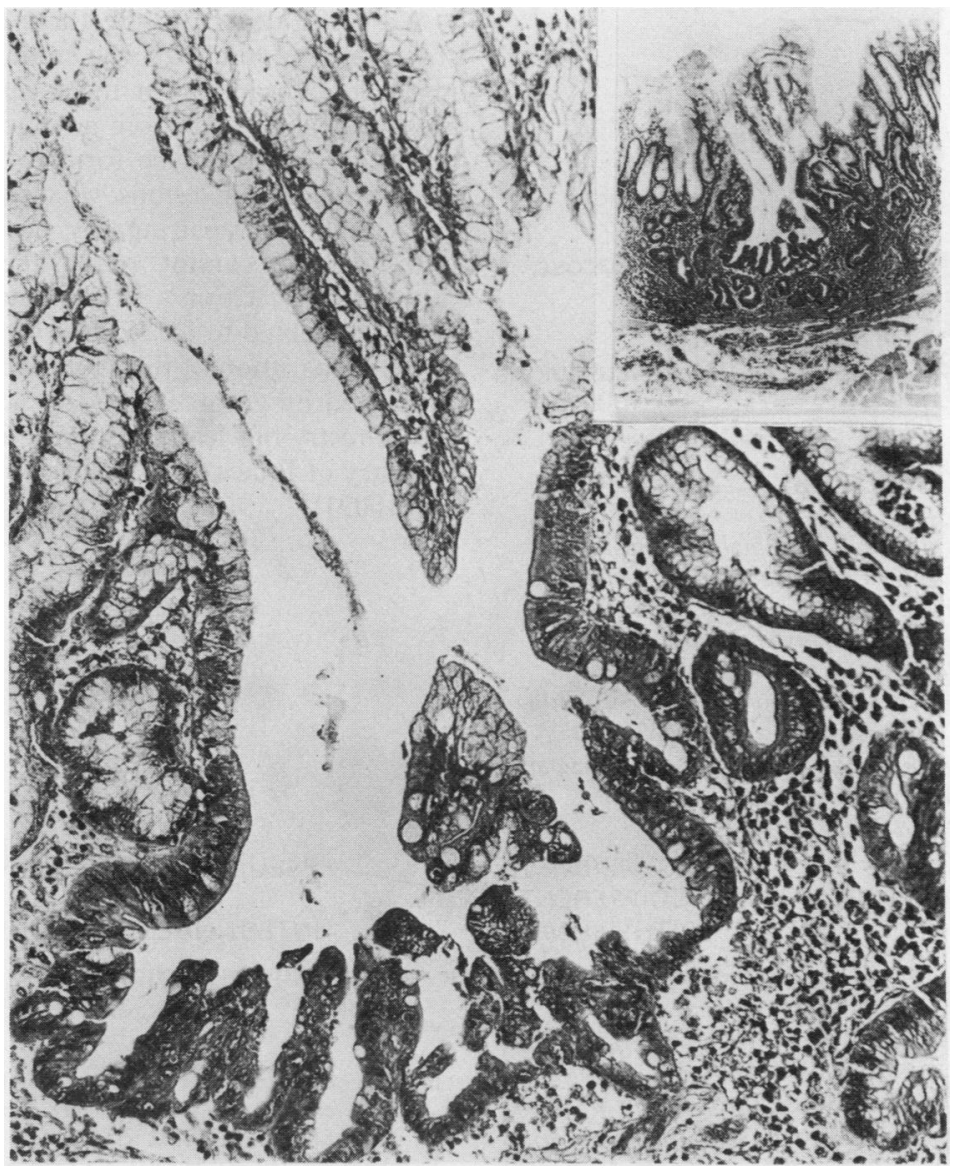

Fig 4 Minute intestinal metaplasia lesion coexisting with typical regenerative undifferentiated epithelium. Transition from regenerative undifferentiated epithelium to intestinal epithelium is recognised at bottom of depression. Original magnification $\times 32$.

membranes were indistinct. This regenerative undifferentiated epithelium was found in 41 of $52(78 \cdot 8 \%)$ minute intestinal metaplasia lesions from the serial sections examined.

\section{Discussion}

Although a large number of epidemiological, ${ }^{6}$ histopathological, ${ }^{1-57}$ and experimental ${ }^{8}$ studies on the intestinal metaplasia of the stomach have been carried out, a three dimensional histopathological study of minute intestinal metaplasia lesions in the stomach has not as far as we know been reported.

The present study shows that 52 minute intestinal metaplasia lesions that had to have arisen from the regenerative process of healing of gastric erosions (fig 5) exhibited the following characteristics: a rough globoid appearance with a depression on the surface three dimensionally; an appreciable reduction in size in the pyloric glands at the centre of the lesions; splitting or fibrous thickening of the muscularis mucosae at the base of the lesion; hyperplasia of the lymphoid tissue between the lesion and adjacent mucosa and in some cases clusters of regenerative foveolar epithelial cells in neighbouring mucosa. It was therefore concluded that these minute intestinal metaplasia lesions originated during the repair process of erosions.

In previous studies on the histogenesis of intestinal metaplasia Moszkowicz suggested that it was the result of incomplete regeneration of surface epithelium which has been repeatedly damaged, and that regenerative undifferentiated epithelium in each healing erosion has the potential to change its differentiation from a gastric to an intestinal pattern. ${ }^{1}$ In later studies some authors thought that intestinal metaplasia might be a reactive change due to tissue 


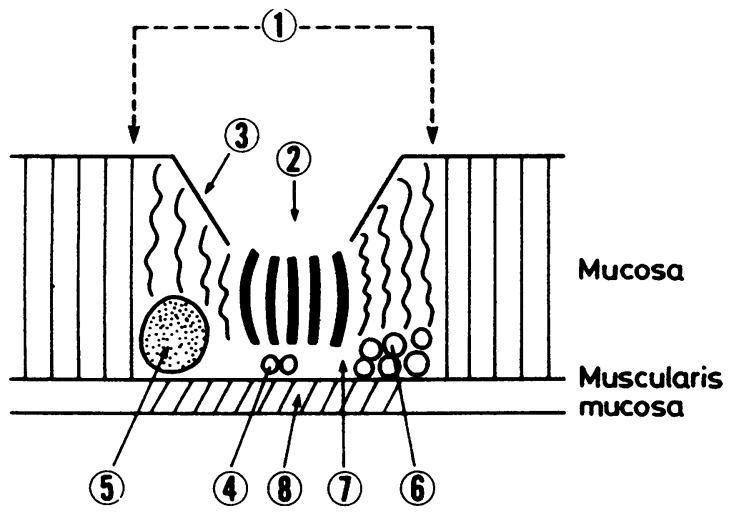

Fig 5 Scheme of typical minute intestinal metaplasia lesion with depression on surface. ( $\mathrm{m}$ : mucosa, $\mathrm{mm}$ : muscularis mucosae) I repairing erosion; 2 minute intestinal metaplasia lesion; 3 hyperplastic regenerative foveolar epithelium; 4 severe decrease of pyloric glands; 5 proliferation of lymphoid tissue; 6 growth of pseudopyloric glands connecting regenerative epithelia; 7 stromal fibrosis and chronic inflammatory cell infiltration of gastic mucosa; 8 splitting and fibrous thickening of muscularis mucosae.
We concluded from the three dimensional reconstruction that most of the minute intestinal metaplasia lesions arising in the regenerative process of erosion healing and more advanced intestinal metaplasia lesions would be formed by an increase and confluence of such lesions, which arose from repeated erosions and repairing by intestinal metaplasia (although we cannot refute the mutation theory proposed by Oota, ${ }^{4}$ Murakami, ${ }^{3}$ and Hattori $^{5}$ ). We are indebted to Dr JR Jass for his kind assistance in the preparation of this manuscript. This study was supported by grants-in-aid for cancer research from the Ministry of Health and Welfare (60-35) and the Ministry of Education, Science and Culture of Japan (59010091).

\section{EROSION $\longrightarrow \begin{aligned} & \text { REGENERATIVE } \\ & \text { UNDIFFERENTIATED } \\ & \text { EPITHELIUM }\end{aligned}$}

Fig 6 Scheme for regeneration of gastric mucosa in cases of gastric erosions.

injury in the course of a disease. ${ }^{9}$ Oohara reported an experimental study in which intestinal metaplasia of the regenerative epithelium was successfully induced in rats simply by treating the gastric mucosa with $0.5 \mathrm{~N}$ sodium hydroxide, and from the histological study of the regenerative epithelial of 549 foci of gastric ulcer it was concluded that regenerative epithelium often gives rise to intestinal metaplasia during the regenerative process. ${ }^{2}$

From this histogenetical study of a minute intestinal metaplasia lesion, it seemed that the lining epithelium in the healing erosion initially takes the form of regenerative undifferentiated epithelium as an expression of regeneration and is then transformed into intestinal epithelium or pseudopyloric gland (fig 6). We could detect minute intestinal metaplasia lesions in which healing erosions and transitions from regenerative undifferentiated epithelium to intestinal epithelium coexisted (fig 5). This finding also supports the above mentioned theory of the histogenesis of intestinal metaplasia.

Okuda reported that multiple minute erosions were often observed endoscopically in the antral mucosa, that metaplastic tubules were discovered only in the depressed mucosa in the early stages, and that in time, these metaplastic foci extended and merged with each other, supporting Moszkowicz's theory. ${ }^{10}$
References

1 Moszkowicz L. Regeneration und Krebsildung an der Magenschleimhaut. Grundlagern einer biologischen Krebstheorie. Arch Kin Chir 1924;132:558-620.

2 Oohara T, Tohma H, Aono G. Intestinal metaplasia of the regenerative epithelia in 549 gastric ulcers. Hum Pathol 1983:14:1066-7.

3 Murakami T, Nakamura S, Suzuki T. A histological study on the mechanism of intestinal epithelial metaplasia in the gastric mucous membrane. Japanese Journal of Cancer Research (GANN) 1955:46:9-14.

4 Oota K. "Metaplastic gastritis". Some consideration of its histogenesis. Japanese Journal of Cancer Research (GANN) 1950;41:72-5.

5 Hattori T. Development of adenocarcinoma in the stomach. Cancer 1986;57:1528-34.

6 Imai T, Murayama H. Time trend in the prevalence of intestinal metaplasia in Japan. Cancer 1983;52:353-61.

7 Morson BC. Carcinoma arising from area of intestinal epithelium in the gastric mucosa. Br J Cancer 1955;9:377-85.

8 Watanabe $\mathrm{H}$. Experimentally induced intestinal metaplasia in the Winster rats by x-ray irradiation. Gastroenterology 1978;75: 796-9.

9 Ming SC, Goldman H, Freiman DG. Intestinal metaplasia and histogenesis of carcinoma in human stomach: light and electron microscopic study. Cancer 1967;20:1418-29.

10 Okuda J, Miyagawa H, Ozaki M. A case of intestinal metaplasia of the stomach showing marked extent for 8 years. Gastroenterological Endoscopy (in Japanese) 1984;26(9):1538-42.

Requests for reprints to: Dr K Mukawa, First Department of Surgery, Gunma University School of Medicine, 3-15-22 Showa-machi, Maebashi 371, Gunma, Japan. 